\title{
DEPRESSÃO E CONSUMO DE ÁLCOOL EM ADOLESCENTES: ANÁLISE DA PRODUÇÃO NO PERÍODO DE OUTUBRO DE 2008 A MARÇO DE 2017
}

\author{
DEPRESSION AND CONSUMPTION OF ALCOHOL IN ADOLESCENTS: ANALYSIS OF PRO- \\ DUCTION IN THE PERIOD OF OCTOBER 2008 TO MARCH 2017
}

\author{
Raul Aragão Martins ${ }^{\mathrm{a}^{*}}$, Camila Fernandes Ribeiro ${ }^{\mathrm{b}^{*}}$, Luciana Aparecida Nogueira Cruz ${ }^{\mathrm{c}^{*}}$ \\ raul@ibilce.unesp.bra ,cami.ribeirof@gmail.com ${ }^{\mathrm{b}}$, lucruz@ibilce.unesp.br \\ Universidade Estadual Paulista - UNESP*

\section{RESUMO}

Introdução: As taxas de depressão e o consumo de álcool entre jovens vêm aumentado significativamente ao longo dos anos. Objetivo: $\mathrm{O}$ presente estudo objetivou identificar a visibilidade deste tema, considerando a produção científica dos últimos 10 anos. Materiais e Método: Trata-se de um estudo de caráter quantitativo que analisou o número de artigos publicados por ano, por revista e de artigos por primeiro autor e coautor em dois ou mais trabalhos. Resultados e Conclusões: Foi observado a escassez de pesquisas que busquem compreender a relação entre o aumento do consumo de álcool e a depressão entre jovens. Desse modo, muito ainda deve ser feito para que seja possível a orientação e melhoria na qualidade de vida dos adolescentes.

Palavras chave: Adolescente, consumo de álcool, depressão

\section{ABSTRACT}

Introduction: Depression rates and alcohol consumption among young people has increased significantly over the years. Objective: This current study aimed to identify the theme visibility, considering the scientific production of the last 10 years. Material and Methods: This is a quantitative study that analyzed the number of articles published per year, per journal and number of articles by first author and co-author in two or more papers. Results and Conclusion: It was observed a scarcity of research that seeks to understand the relationship between increased alcohol consumption and depression among young people. Thus, there is still much more to change to make it possible to guide adolescents and improve their life quality.

Keywords: Adolescent, alcohol consumption, depression 


\section{Introdução}

Atualmente, uma das maiores preocupações dos pais e dos professores, em termos de saúde, é o crescente consumo de álcool entre adolescentes. O álcool é a droga mais consumida em todo mundo, acarretando um dos maiores problemas de saúde pública no Brasil ${ }^{1}$, já que foi estimado um crescimento de $70,4 \%$ no consumo de álcool no país. Isso deixa o maior país sul-americano entre os 25 países que mais consomem bebidas alcoólicas ${ }^{2}$.

Em uma pesquisa realizada com 834 alunos de ensino médio de uma escola estadual da zona urbana e rural, foi observado que o início do consumo de álcool ocorre precocemente entre os jovens, sendo que $80,8 \%$ dos estudantes entrevistados já fizeram uso de álcool ${ }^{3}$, corroborado por outros estudos também ${ }^{4}$. Além disso, a média de idade para o primeiro consumo se encontra entre 12 e 13 anos de idade e $99 \%$ dos entrevistados experimentaram algum tipo de bebida alcoólica antes dos 18 anos $^{5-7}$. Ademais, viu-se que, em cerca de $33 \%$ dos casos, a bebida foi oferecida por parentes ou amigos ${ }^{5}$, mostrando a influência exercida sob o jovem pelo grupo social do qual ele faz parte.

É possível verificar a importância dos estudos sobre o uso de álcool entre adolescentes em várias pesquisas que mostram que o índice de consumo entre eles vem aumentando consideravelmente, acarretando problemas de saúde e acidentes externos ${ }^{3,7-8}$.

O álcool é uma substância importante em contextos sociais diversos, tanto alegres quanto de sofrimento, uma vez que auxilia na liberação das emoções e propicia interação com o grupo e sensação de bem-estar momentâneo'. Essas situações e fatos podem estar ligadas ao aumento do consumo de bebidas entre jovens, pois eles se encontram em uma fase de transição para a vida adulta, o que, aliado a um relacionamento insatisfatório com a família e ao livre acesso à essa substância ${ }^{4}$, acarreta muitas incertezas e conflitos.

Um dos maiores responsáveis por problemas comuns na adolescência, como brigas, término de relacionamentos, lesões, entre outros, é o consumo excessivo da bebida alcoólica ${ }^{2}$. Alguns problemas de saúde também são potencializados por esse uso abusivo, como é o caso da depressão ${ }^{2}$.
O álcool é uma droga depressora do sistema nervoso central (SNC), dessa forma, ele causa uma sensação de euforia inicial, seguida de uma diminuição da atividade do SNC e da atividade motora, podendo agravar consideravelmente o quadro depressivo do indivíduo ${ }^{2}$.

De acordo com o Manual Diagnóstico e Estatístico de Transtornos Mentais ${ }^{10}$, os transtornos depressivos são caracterizados, na adolescência, por irritabilidade e instabilidade de humor, perda de energia, apatia e desinteresse, retardo psicomotor, alterações de apetite e peso, isolamento social e dificuldade de concentração. Esses sintomas podem acarretar baixo desempenho escolar, baixa autoestima, tentativas de suicídio e problemas de comportamento, principalmente quando associado ao uso abusivo do álcool e de outras drogas ${ }^{10}$.

Um estudo recente, em nível nacional, com 75 mil estudantes entre 12 e 17 anos, demonstrou que 1/3 dos adolescentes apresenta algum tipo de sofrimento psíquico, como ansiedade e depressão, sendo mais comum entre meninas mais velhas (entre 15 e 17 anos $^{11}$ ), conforme relatado em outros estudos ${ }^{12}$. Esses números podem estar relacionados ao período de transição da adolescência para a vida adulta, em que esses jovens se encontram, com suas diversas mudanças físicas, mentais e sociais.

Embora ainda não tenha sido possível estabelecer uma relação exata entre os índices de depressão e o consumo de álcool ${ }^{13}$, é observado um aumento considerável na frequência com que essa doença atinge os jovens ${ }^{14}$ concomitantemente com o já relatado aumento do consumo de bebidas alcoólicas. Sendo assim, é extremamente necessário um olhar mais atento para essas questões, e, embora haja um aparente aumento dessa preocupação, a pouca quantidade de artigos científicos publicados acerca das temáticas depressão e consumo de bebida alcoólica, bem como a relação entre elas, evidencia o quanto ainda é preciso ser debatido e pesquisado.

Pensando nisso, atualmente, a forma mais comum de disseminação de conhecimento científico é a publicação em revistas cientificas, sejam elas online ou impressas. A importância da análise das produções científicas, bem como das citações dos autores e da avaliação das revistas, 
é pautada na necessidade de saber não só como o conhecimento científico vem sendo divulgado ao público, mas também qual a qualidade $\mathrm{e}$ visibilidade dos assuntos propostos ${ }^{15}$.

Dessa forma, os estudos bibliométricos são uma ferramenta importante de avaliação dessas produções, uma vez que, aliados a outros instrumentos, mensuram como o conhecimento vem sendo difundido, o impacto dos autores, qual pensamento vigora entre os acadêmicos, podendo assim, gerenciar, de maneira mais eficaz, as publicações científicas e identificar as metodologias e teorias bem estabelecidas ${ }^{15}$.

Nesse sentido, o presente estudo buscou analisar a produção científica existente no período de outubro de 2008 a março de 2017 acerca do assunto sob a justificativa de avaliar qual a visibilidade dada a este tema. A partir disso, seria ressaltada a importância e a necessidade de discussão sobre os transtornos depressivos e o consumo de álcool que comumente afetam os adolescentes.

Este trabalho se faz importante pela necessidade de se estudar e compreender um segmento da população muitas vezes menosprezado, os adolescentes, uma vez que há uma escassez de pesquisas científicas voltadas à pesquisa da saúde física e mental desse grupo.

\section{Desenvolvimento}

A metodologia deste estudo consistiu em um levantamento bibliográfico, utilizando a plataforma online www.bvs-psi.org.br. Para isso, foi feita uma análise da produção científica referente ao tema "depressão e consumo de álcool entre adolescentes". Para a localização dos artigos na base de dados, foram utilizadas as seguintes palavras: "depressão", "consumo de álcool", "adolescentes".

Os parâmetros bibliométricos utilizados para este estudo foram:

a) Quantidade de artigos por ano, entre o período de outubro de 2008 e março de 2017;

b) Quantidade de artigos publicados por revista e sua respectiva classificação da Capes no quadriênio 2013-2016 nas áreas de avaliação de Educação e Ensino; c) Quantidade de artigos publicados por autor, levando-se em consideração o primeiro autor de cada artigo.

Justifica-se a escolha das áreas de avaliação Educação e Ensino pela necessidade de entender que os aspectos emocionais e físicos dos adolescentes estão intimamente ligados com a área educacional, uma vez que a escola tem papel atuante na formação cidadã e consciente dos estudantes. Dessa forma, verifica-se a importância desses assuntos serem pesquisados e discutidos nas áreas citadas anteriormente.

A amostra possui um total de 24 artigos selecionados de acordo com os objetivos desta pesquisa durante o período de 10 anos (20082017).

Para avaliar o impacto das publicações, este estudo considerou o Qualis de cada periódico em que os artigos foram publicados. Para tal finalidade, foi utilizada a Plataforma Sucupira (https://sucupira.capes.gov.br ${ }^{16}$ ), disponibilizada online pela Capes, e foi considerada a classificação dos periódicos nas áreas de Educação e Ensino.

No que diz respeito à autoria das publicações, foi levado em consideração o primeiro autor de cada artigo e também coautores que se destacaram por estarem em colaboração com mais de um trabalho.

A partir da análise das publicações científicas disponíveis na plataforma online www. bvs-psi.org.br, foi possível identificar que, nos 10 anos analisados, foram poucas as publicações sobre o tema em questão, com um total de apenas 24 artigos, contrariando as expectativas iniciais desta pesquisa acerca da disponibilidade de informações científicas pertinentes a respeito do tema. A figura 1 mostra uma produção média de 2,4 artigos ano, mas com a metade do período com somente uma publicação por ano. A média teve um incremento por nos anos de 2011 e 2012 terem sido publicados cinco e sete artigos, respectivamente. 
Figura 1 - Quantidade de artigos publicados por ano

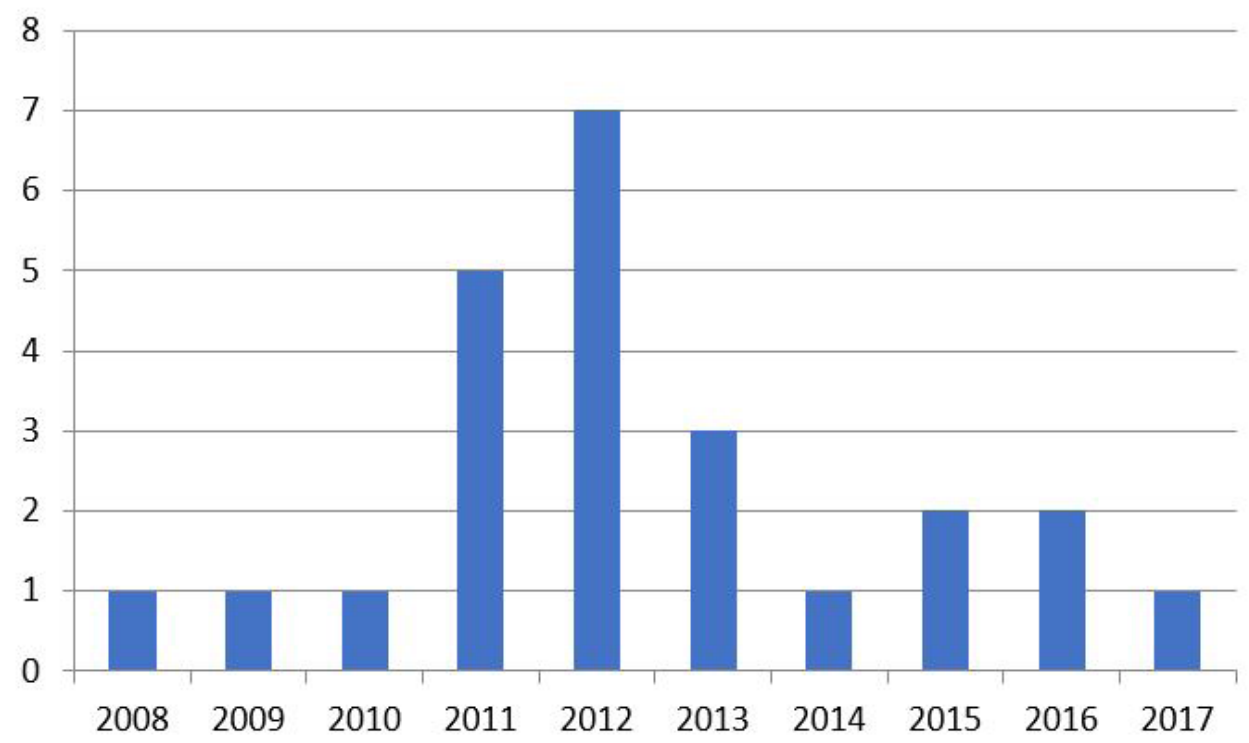

Referente ao impacto das publicações, levando-se em consideração o Qualis de cada periódico em que os artigos foram publicados, nas áreas de Educação e Ensino, foram consultados 16 periódicos, dos quais 14 apresentaram apenas

um artigo publicado. As exceções foram a revista Ciência \& Saúde Coletiva e a Revista Eletrônica Saúde Mental Álcool e Drogas, com 2 e 8 publicações respectivamente (Quadro 1).

Quadro 1 - Quantidade de artigos publicados por revista e sua respectiva classificação da Capes (Qualis) no quadriênio 20132016 nas áreas de avaliação de Educação e Ensino. (Disponível em: https://sucupira.capes.gov.br)

\begin{tabular}{|c|c|c|c|}
\hline Revista & Quantidade & $\begin{array}{l}\text { Qualis na área de } \\
\text { Educação }\end{array}$ & $\begin{array}{l}\text { Qualis na área de } \\
\text { Ensino }\end{array}$ \\
\hline Acta Paulista de Enfermagem & 1 & A1 & B3 \\
\hline Cadernos de Saúde Coletiva & 1 & Sem classificação & Sem classificação \\
\hline Cadernos de Saúde Pública & 1 & $\mathrm{~B} 1$ & $\mathrm{~A} 2$ \\
\hline $\begin{array}{l}\text { Cadernos de Terapia } \\
\text { Ocupacional da UFSCar }\end{array}$ & 1 & B5 & B4 \\
\hline Ciência \& Saúde Coletiva & 2 & $\mathrm{~A} 2$ & A1 \\
\hline Paidéia & 1 & A1 & Sem classificação \\
\hline Psicologia \& Sociedade & 1 & $\mathrm{~A} 2$ & $\mathrm{~A} 2$ \\
\hline Psicologia: Ciência e Profissão & 1 & B1 & B1 \\
\hline Psicologia: Reflexão e Crítica & 1 & A1 & $\mathrm{B} 2$ \\
\hline Psicologia: Teoria e Prática & 1 & B1 & Sem classificação \\
\hline $\begin{array}{l}\text { Revista Baiana de } \\
\text { Pública }\end{array}$ & 1 & Sem classificação & Sem classificação \\
\hline Revista Brasileira de Psiquiatria & 1 & B1 & Sem classificação \\
\hline Revista Gaúcha de Enfermagem & 1 & B1 & B3 \\
\hline Revista de Psiquiatria Clínica & 1 & Sem classificação & Sem classificação \\
\hline Saúde e Sociedade & 1 & $\mathrm{~A} 2$ & A1 \\
\hline
\end{tabular}




\begin{tabular}{|l|c|c|c|}
\hline SMAD - Revista Eletrônica & 8 & Sem classificação & B1 \\
Saúde Mental Álcool e Drogas & & & \\
\hline
\end{tabular}

Um fato importante a ser mencionado é que nenhum periódico obteve uma classificação inferior a $\mathrm{B} 4 \mathrm{em}$ ambas as áreas, sendo a maioria classificada como A1, A2, B1 e B2 (Quadro 1).

Para avaliar a autoria das publicações, levouse em consideração o primeiro autor de cada artigo e coautores que evidenciaram estar em colaboração com mais de um trabalho. Dessa forma, observou-se que, embora alguns autores apresentassem apenas um artigo de sua autoria, existiam colaboradores que estavam presentes em publicações diferentes, indicando assim uma possível equipe de trabalho relacionada ao tema em questão (Quadro 2).
A maior produtividade se encontra na coautoria de Coutinho, M. P. L., com quatro artigos, seguida dos coautores Carolino, Z. C. G. e Martins, R. A., com dois artigos cada um. Ribeiro, K. C. S. apresenta dois artigos em primeira autoria e um como coautor (Quadro 2). O que é bastante interessante e relevante a essa pesquisa é o fato de que entre as quatro publicações de Coutinho, M. P. L., a coautora mais recorrente entre os artigos analisados, três delas são em parceria com os pesquisadores Carolino, Z. C. G. e Ribeiro, K. C. S., tendo os outros dois autores identificados apresentado uma produtividade elevada em comparação ao restante (Quadro 2).

Quadro 2 - Quantidade de artigos publicados por autor levando-se em consideração autoria e mais de uma publicação com coautoria.

\begin{tabular}{|c|c|c|}
\hline Autor & Primeiro autor & Coautor \\
\hline Anjos, K. F. ${ }^{(17)}$ & 1 & - \\
\hline Aragão, T. A. ${ }^{(18)}$ & 1 & - \\
\hline Avanci, J. Q. ${ }^{(19)}$ & 1 & - \\
\hline Campos, J. A. D. B. ${ }^{(20)}$ & 1 & - \\
\hline Carolino, Z. C. G. ${ }^{(22,31)}$ & - & 2 \\
\hline Coutinho, M. P. L. ${ }^{(18,22,30-31)}$ & - & 4 \\
\hline Dallo, L. ${ }^{(21)}$ & 1 & - \\
\hline Damião, N. F. ${ }^{(22)}$ & 1 & - \\
\hline Figueiredo, T. C. ${ }^{(4)}$ & 1 & - \\
\hline Freitas, E. A. M. ${ }^{(23)}$ & 1 & - \\
\hline Garcia Del Castillo, J. A. ${ }^{(24)}$ & 1 & - \\
\hline Giacomozzi, A. I. ${ }^{(25)}$ & 1 & - \\
\hline Hernandez-Cortaza, B. A. ${ }^{(26)}$ & 1 & - \\
\hline Locatelli, D. ${ }^{(5)}$ & 1 & - \\
\hline Martins, R. A. ${ }^{(13,21)}$ & - & 2 \\
\hline Mendez-Ruiz, M. D. ${ }^{(8)}$ & 1 & - \\
\hline Pasuch, C. (27) & 1 & - \\
\hline Pereira, M. O. ${ }^{(28)}$ & 1 & - \\
\hline Portugal, F. B. (29) & 1 & - \\
\hline Pratta, E. M. M..$^{(9)}$ & 1 & - \\
\hline
\end{tabular}




\begin{tabular}{|l|l|l|}
\hline Ribeiro, K. C. S. $^{(22,30-31)}$ & 2 & 1 \\
\hline Rodriguez Puente, L. A. $^{(32)}$ & 1 & - \\
\hline Salle, E. $^{(12)}$ & 1 & - \\
\hline Teixeira, P. S. $^{(13)}$ & 1 & - \\
\hline Teixeira, C. C. $^{(33)}$ & 1 & - \\
\hline Veiga, L. D. B. $^{(3)}$ & 1 & - \\
\hline
\end{tabular}

Foi feito um levantamento dos principais aspectos que deveriam ser enfocados nos artigos selecionados e seguiu-se dessa maneira: objetivos, sujeitos pesquisados, instrumentos utilizados e resumo dos resultados. Feito esse panorama geral da análise dos artigos, foi possível observar um padrão nos resultados apresentados pelos artigos tanto para o consumo de álcool, em que é verificado um aumento considerável no uso das bebidas e uma diminuição da idade em que se inicia, quanto aos índices crescentes de depressão entre os adolescentes.

Os artigos analisados foram agrupados em 4 seções de acordo com o objetivo central: a) depressão ${ }^{12,18,19,22,30,31}$; b) depressão e drogas $\mathrm{s}^{13,26,33}$; c) epidemiologia álcool e outras $\operatorname{drogas}^{3-5,8,9,17,20,21,23-25,28,32}$; d) uso de álcool e de outras drogas ${ }^{27,29}$.

Os trabalhos do primeiro grupo tiveram seus objetivos centrados, de uma forma geral, em identificar os fatores de risco que levam os estudantes à depressão e também em apreender as representações sociais da depressão entre esses jovens. Os sujeitos pesquisados são jovens de ambos os sexos, matriculados no ensino médio, oriundos de escolas públicas e privadas, e apenas um desses artigos selecionou também alunos de ensino fundamental para compor a pesquisa ${ }^{19}$. De maneira geral, os instrumentos mais utilizados para alcançar os objetivos das pesquisas foram: Inventário de Depressão Infantil (CDI), Inventário de Depressão de Beck (BDI) e Teste de Associação Livre de Palavras (TALP).

Os resultados apresentados indicaram que as representações sociais da depressão, para os adolescentes, são sinônimo de dor, mágoa, infelicidade, morte e ideias suicidas, desânimo, angústia, solidão e choro. Além disso, adolescentes que apresentam algum tipo de conflito como baixa autoestima, tristeza, dificuldade de relacionamento social, desilusão amorosa, estrutura familiar menos preservada, como a composta por padrasto/ madrasta ou monoparentais, e por fim o consumo de álcool e outras substâncias, mostram-se mais vulneráveis aos sintomas de depressão.

O segundo grupo analisado, "depressão e drogas", pautou principalmente seu objetivo em identificar a relação entre o consumo de álcool e a depressão entre os adolescentes. Os sujeitos selecionados para compor as pesquisas foram exclusivamente estudantes matriculados no ensino médio, de ambos os sexos. Um fato importante a ser levado em consideração é que um dos trabalhos foi realizado em uma escola pública em Veracruz, no México. Os instrumentos utilizados em comum foram: BDI e Alcohol Use Disorders Identification Test (AUDIT). Dos três trabalhos analisados nessa seção, apenas o que foi realizado no México indica que a maioria dos jovens que consomem álcool apresentam algum nível de depressão.

O grupo denominado "epidemiologia álcool e outras drogas", que apresenta o maior número de artigos, tem como objetivos principais identificar os fatores associados ao uso de bebidas alcoólicas e o padrão de consumo entre os estudantes. As pesquisas selecionaram alunos de ambos os sexos matriculados no ensino médio, sendo que um dos artigos utilizou alunos de ensino fundamental ${ }^{25}$. Vale ressaltar que dois artigos foram realizados em Nuevo León, no México. De uma forma geral, o instrumento mais utilizado foi o AUDIT, seguido pelo questionário autoaplicável validado pelo Centro Brasileiro de Informação sobre Drogas Psicotrópicas (Cebrid).

Os resultados apresentados pelas pesquisas trazem, de forma quase unânime, a constatação de que os sujeitos pesquisados já consumiram álcool de forma precoce. Além disso, foi possível observar a forte influência de amigos e 
familiares para o início dessa prática por ser uma droga lícita e socialmente aceita e amplamente difundida. Também é constatado que a média para o início do consumo é de 13 anos e muitos jovens continuam fazendo uso dessa substância, evidenciando a alta prevalência do consumo, podendo levar a dependência. Vale ressaltar que o álcool é a droga mais consumida entre os adolescentes e a motivação para seu consumo se pauta na busca pela diversão, inserção em determinado grupo social, falta de diálogo com a família, fuga dos problemas e ansiedades comuns nessa faixa etária.

Por fim, o último grupo é composto por artigos de revisão que buscam conhecer e verificar a prevalência do uso de drogas entre os estudantes. As revisões foram feitas com artigos de 1999 até 2012, com enfoque em alunos do ensino fundamental e médio e, para isso, foram utilizadas bases de dados como SciELO, MEDLINE, LILACS, entre outros. Por meio dessas análises, foi observado um crescimento no uso de drogas entre os estudantes somado a um contato precoce com tais substâncias. Além disso, eles indicaram uma alta prevalência de uso de álcool e tabaco, demonstrando que as drogas lícitas são mais presentes na vida dos adolescentes.

Embora as revistas analisadas se mostrem positivas quanto seu impacto na área de Ensino e Educação, mostrando-se úteis a comunidade acadêmica e a população, os dados apresentados neste trabalho evidenciam a necessidade de se levantar o questionamento do porquê a depressão e o consumo de álcool entre os adolescentes, embora tão preocupante e alarmante, ainda é esquecido entre os pesquisadores,

No entanto, foi possível observar um indicativo da existência de um grupo de pesquisa estabelecido, com interesse em estudar fatores importantes que influenciam a saúde de adolescentes. Analisando os quatro autores citados anteriormente, na seção 3 , vemos um padrão de estudo que poderia separá-los, superficialmente, e apenas para fins de compreensão, em dois grupos: o primeiro composto por Coutinho, M. P. L., Carolino, Z. C. G. e Ribeiro, K. C. S. e o segundo composto por Martins, R. A. O. O primeiro, fazendo uma leitura apenas do título dos artigos, busca pesquisar mais especificamente transtornos depressivos em adolescentes, enquanto o segundo tem um foco maior no consumo de álcool. Contudo, a relação entre eles é de extrema importância para entendermos melhor a dinâmica de como esses dois fatores agem e influenciam a vida e a saúde física e mental dos adolescentes, uma vez que os níveis de crescimento de ambos são alarmantes ${ }^{11,14}$.

A fase da adolescência é marcada pela transição da vida infantil para a vida adulta, o que traz consigo uma série de fatores significativos no desenvolvimento do jovem, como as mudanças corporais e hormonais, mudanças no comportamento e a formação de caráter e personalidade $^{33}$. É nessa fase também que as influências externas atuam com maior pressão na construção da identidade do indivíduo, como a cultura e os valores ${ }^{29,33}$. Assim, verifica-se a importância dos grupos sociais e da família aos quais esses jovens estão vinculados, pois estudos demonstram que o primeiro contato do jovem com o álcool, por exemplo, é no ambiente familiar e entre amigos ${ }^{5,28}$.

Um fato importante a ser discutido é que muitos jovens com sintomatologia depressiva não se encontram tristes o tempo todo, mas irritáveis e com humor instável, levando a comportamentos destrutivos. Uma característica importante da adolescência é a tendência de se expressar por meio de ações concretas e não por palavras e conversas, desse modo, os adolescentes buscam solucionar seus problemas de maneira rápida, recorrendo muitas vezes ao uso de drogas ${ }^{22}$, sejam elas lícitas ou ilícitas, e, nesse momento, o álcool se mostra um forte aliado $^{26}$, uma vez que é legalizado e comercializado sem grandes dificuldades e é amplamente aceito socialmente.

O processo da construção da identidade é algo de fato complexo e, muitas vezes, conflituoso. Assim, o álcool funciona como uma válvula de escape para o enfrentamento dos problemas da adolescência, pois o jovem busca um alívio para suas angústias. Esse caminho pode ter então duas consequências: o abuso do consumo de álcool e a depressão ${ }^{26}$. Em vista disso, é necessário que haja a construção de um vínculo de apoio entre esses jovens, suas famílias, as instituições de ensino e os sistemas de saúde, que, por meio de estratégias de educação que entendam a realidade desses jovens, os oriente de forma adequada. 
Por fim, vale ressaltar que, a partir da análise feita sob o panorama geral dos artigos analisados, é possível demonstrar a importância do estudo desses temas e também da relação entre eles, uma vez que ainda não é de fato conhecido se o consumo do álcool leva a depressão ou o contrário. Sabendo dessa informação com mais clareza, é possível aplicar medidas de prevenção e também de cuidados com esses estudantes.

Para que isso ocorra, é preciso que esse jovem seja visto e que a sua realidade seja conhecida e compreendida, sendo neste sentido que este estudo procura contribuir. Por meio deste trabalho, foi possível observar uma escassez de pesquisas científicas dispostas a entender esse problema de saúde pública que é o aumento do consumo de álcool e das taxas de depressão entre os adolescentes, ao contrário do que se esperava.

\section{Conclusão}

Estudos que visem à avaliação da saúde física e mental dos jovens, no que diz respeito ao consumo de álcool e as taxas de depressão, se fazem necessários, uma vez que é nessa fase do desenvolvimento humano que ocorre uma importante mudança: a transição da infância para a vida adulta. É nesse período que o jovem se depara com uma liberdade que antes lhe era distante, e assim, comportamentos comumente feitos por adultos são imitados, como o caso do consumo de bebidas alcoólicas.

No entanto, é possível perceber que nessa fase há um outro fator importante a ser considerado: os problemas emocionais enfrentados por esses adolescentes. Problemas esses que podem ser relacionados às dificuldades de adaptação social, não aceitação pessoal, baixa autoestima, entre outros aspectos, que induzem não só o consumo de álcool, mas também podem levar à transtornos depressivos significativos.

Desse modo, se faz extremamente necessário compreender a possível relação entre esse aumento alarmante do consumo de bebidas alcoólicas conjuntamente com o aumento significativo de transtornos depressivos entre os jovens, pois esse é um problema de saúde pública que deve ter a devida atenção, visando à melhoria da qualidade de vida desse grupo em questão em conjunto com os sistemas de saúde, as instituições de ensino e a família, na busca de aconselhamentos e orientações sobre como lidar com as situações conflituosas da vida sem recorrer a drogas ou adoecerem.

\section{Referências}

1. Ministério da Saúde. A política do ministério da saúde para a atenção integral a usuários de álcool e outras drogas [Internet]. Brasília: Ministério da Saúde; 2003 (citado em 11 set 2017) Disponível em: http://bvsms. saude.gov.br/bvs/publicações/pns_alcool_drogas.pdf

2. Cruz LAN, Martins RA \& Silva IA. Meus alunos estão bebendo! E agora?: guia teórico-prático para educadores sobre intervenção breve para reduzir o consumo de álcool entre estudantes. $1^{\text {a }}$ edição. Curitiba: Editora Prismas, $2016.130 \mathrm{p}$.

3. Veiga LDB, Santos VC, Santos MG, Ribeiro JF, Amaral ASN, Nery AA, et al. Prevalência e fatores associados à experimentação e ao consumo de bebidas alcoólicas entre adolescentes escolares. Cad. saúde colet., Rio de Janeiro. 2016. Set, 24(3):368-375.

4. Figueiredo TC, Freitas RM. Socio-demographic profile and the licit and illicit drugs use among secondary education students. SMAD, Rev. Eletrônica Saúde Mental Álcool Drog. (Ed. port.), Ribeirão Preto. 2013. Abr, $9(1): 3-10$.

5. Locatelli D, Sanchez Z, Opaleye E, Carlini C, Noto A. Socioeconomic influences on alcohol use patterns among private school students in São Paulo. Rev. Bras. Psiquiatr., São Paulo. 2012. Jun, 34(2):193-200.

6. Martins RA, Manzato AJ, Cruz LAN. O uso de bebidas alcoólicas entre adolescentes. Rio de Janeiro: NAU Editora: FAPERJ. 2005. p. 301-326.

7.

8. Vieira DL, Ribeiro M, Romano M, Laranjeira RR. Álcool e adolescentes: estudo para implementar políticas municipais. Rev. Saúde Pública, São Paulo, 2007. Jun 41(3):396-403.

9. Mendez-Ruiz, MD, Alonso-Castillo MTJ, AlonsoCastillo MM, Uribe-Alvarado JI, Armendáriz-Garcia NA. Relationship between risk perception and alcohol consumption in adolescents. SMAD, Rev. Eletrônica Saúde Mental Álcool Drog. (Ed. port.), Ribeirão Preto. 2015. Set 11(3):161-167. 
10. Pratta EMM, Santos MA. Risk factors for alcohol use in the life and in the year among high school teenagers. SMAD, Rev. Eletrônica Saúde Mental Álcool Drog. (Ed. port.), Ribeirão Preto. 2013. Abr 9(1):18-24.

11. DSM-V. Manual Diagnóstico e Estatístico de Transtornos Mentais. 5a edição. Porto Alegre: Artes Médicas, 2014. $992 \mathrm{p}$.

12. Lopes CS, Abreu GA, Santos DF, Menezes PR, Carvalho $\mathrm{KMB}$, Cunha CF, et al. Erica: Prevalence of common mental disorders in Brazilian adolescents. Revista de Saúde Pública. 2016. 50(supl 1):1-14s.

13. Salle E, Rocha NS, Rocha TS, Nunes C, Chaves MLF. Escalas psicométricas como instrumentos de rastreamento para depressão em estudantes do ensino médio. Rev. psiquiatr. clín., São Paulo. 2012. 39(1):24-27.

14. Teixeira PS, Stefanini MCB, Martins RA, Cruz LAN. Desenvolvimento cognitivo e sintomas depressivos em adolescentes que fazem uso de bebidas alcoólicas. SMAD - Revista Eletrônica Saúde Mental Álcool e Drogas, Ribeirão Preto. 2011. Abr 7(1):03-09.

15. Bahls SC. Depressão na adolescência: características clínicas. Rev Interação Psicol. 2002. 6(1):49-57.

16. Beuren IM, da Silva MZ Características bibliométricas dos artigos sobre gestão hospitalar publicados em periódicos de alto impacto. Rev. cuba. inf. cienc. salud, La Habana.2014. Mar 25(1):36-65.

17. Brasil. Plataforma Sucupira [Internet]. 2016 (citado em 4 out 2017). Disponível em https://sucupira. capes.gov.br/sucupira/public/consultas/coleta/ veiculoPublicacaoQualis/listaConsultaGeralPeriodicos. jsf

18. Anjos KF, Santos VC, Almeida OS. Caracterização do consumo de álcool entre estudantes do ensino médio. Revista Baiana de Saúde Pública, [S.l.]. 2013. Fev 36(2):418.

19. Aragão TA, Coutinho MPL, Araujo LF, Castanha AR. Uma perspectiva psicossocial da sintomatologia depressiva na adolescência. Ciênc. Saúde coletiva, Rio de Janeiro, 2009. Abr 14(2):395-405.

20. Avanci JQ, Assis SG, Oliveira RVC. Sintomas depressivos na adolescência: estudo sobre fatores psicossociais em amostra de escolares de um município do Rio de Janeiro, Brasil. Cad. Saúde Pública, Rio de Janeiro. 2008. Out 24(10):2334-2346.

21. Campos JADB, Almeida JC, Garcia PPNS, Faria JB. Consumo de álcool entre estudantes do ensino médio do município de Passos - MG. Ciênc. saúde coletiva, Rio de Janeiro. 2011. Dez 16(12):4745-4754.
22. Dallo L, Martins RA. Uso de álcool entre adolescentes escolares: um estudo-piloto. Paidéia (Ribeirão Preto). Universidade de São Paulo, Faculdade de Filosofia Ciências e Letras de Ribeirão Preto. 2011. 21(50):329334, 2011.

23. Damião NF, Coutinho MPL, Carolino ZCG, Ribeiro KCS. Representações sociais da depressão no ensino médio um estudo sobre duas capitais. Psicologia \& Sociedade. 2011. 23(1):114-124.

24. Freitas EAM, Luis MAV. Perception of students about alcohol consumption and illicit drugs. Acta paul. enferm., São Paulo. 2015. Ago 28(5):408-414.

25. Garcia Del Castillo JA, Dias PC, Castelar-Perim P. Autorregulação e consumo de substâncias na adolescência. Psicol. Reflex. Crit., Porto Alegre. 2012. 25(2):238-247.

26. Giacomozzi AI, Itokasu MC, Luzardo AR, Figueiredo CDS, Vieira M. Levantamento sobre uso de álcool e outras drogas e vulnerabilidades relacionadas de estudantes de escolas públicas participantes do programa saúde do escolar/saúde e prevenção nas escolas no município de Florianópolis. Saude soc., São Paulo. 2012. Set 21(3):612 622.

27. Hernandez-Cortaza BA, Cortaza-Ramirez L, CostaJunior ML. Consumption of alcohol and depression in students of a public school of Coatzacoalcos, Veracruz, Mexico. SMAD, Rev. Eletrônica Saúde Mental Álcool Drog. (Ed. port.), Ribeirão Preto. 2012. Dez 8(3):142-147.

28. Pasuch C, Oliveira MS. Levantamento sobre o uso de drogas por estudantes do ensino médio: Uma revisão sistemática. Cad. Ter. Ocup. UFSCar, São Carlos. 2014. 22(Suplemento Especial):183-195.

29. Pereira MO, Silva SS, Oliveira MAF, Vargas D, Colvero LA, Leal BMML. The perception of adolescents about alcohol and other drugs in the family context. SMAD, Rev. Eletrônica Saúde Mental Álcool Drog. (Ed. port.), Ribeirão Preto. 2011. Dez 7(3):148-154.

30. Portugal FB, Siqueira MM. Use of psychoactive substances by students of primary and secondary education: a systematic review from 1999 to 2009. SMAD, Rev. Eletrônica Saúde Mental Álcool Drog. (Ed. port.), Ribeirão Preto. 2012. Dez 8(3):155-163.

31. Ribeiro KCS, Coutinho MPL, Nascimento ES. Representação social da depressão em uma Instituição de Ensino da Rede Pública. Psicol. cienc. prof., Brasília. 2010. Set 30(3):448-463. 
32. Ribeiro KCS, Medeiros CS, Coutinho MPL, Carolino ZCG. Representações sociais e sofrimento psíquico de adolescentes com sintomatologia depressiva. Psicol. teor. prat., São Paulo. 2012. Dez 14(3):18-33.

33. Rodriguez Puente LA, Alonso-Castillo BA, AlonsoCastillo MM, Alonso-Castillo MT, Armendáriz-Garcia NA Olivia-Rodriguez NN. Consumption of alcohol and tobacco in adolescents. SMAD, Rev. Eletrônica Saúde Mental Álcool Drog. (Ed. port.), Ribeirão Preto. 2016. Dez 12(4):200-206.

34. Teixeira CC, Guimarães LSP, Echer IC. Fatores associados à iniciação tabágica em adolescentes escolares. Rev Gaúcha Enferm.2017. Mar 38(1):e69077.

Como citar este artigo:

Martins RA, Ribeiro CF, Cruz LAN. Análise de produção científica sobre depressão e consumo de álcool em adolescentes. Rev. Aten. Saúde. 2019;17(60):91-100 